\title{
Editoriale \\ Tempo di framework. Non sprechiamolo...
}

\author{
Alberto Quagli
}

Nel recente gergo contabile italiano l'espressione Framework è entrata in voga dal 1989, anno in cui lo IASC diffuse la versione del Framework che è ancora in vigore. Il framework rappresenta in sostanza la fonte di concetti basilari ai quali si devono ispirare tutti gli standard riferiti alle specifiche poste contabili e alle problematiche di contabilizzazione delle singole operazioni.

Avendo per riferimento il Framework dello IASB, il contenuto di tale documento basilare comprende le finalità del bilancio, strettamente connesse con la definizione degli utenti e delle relative esigenze informative, le assunzioni di base, i postulati dell'informazione contabile (caratteristiche qualitative che determinano l'utilità dell'informativa contenuta nel bilancio), gli elementi componenti il bilancio, i concetti fondamentali di attività, di passività e di patrimonio netto, di costo e di ricavo, i criteri generali di contabilizzazione e valutazione, il concetto di capitale e della sua relativa conservazione.

Dal 2005 circa, sul Framework contabile si è riacceso il dibattito. Da quel periodo IASB e FASB stanno procedendo, non senza qualche rallentamento, alla stesura di un nuovo Framework che condizionerà il futuro di buona parte della contabilità mondiale. Delle sette fasi di cui si compone l'ambizioso progetto, ad oggi solo la prima si può dire conclusa (Objectives and qualitative characteristics), le tre successive sono in corso: (B) Definitions of elements, recognition and derecognition, (C) Measurement, (D) Reporting entity concept. Le rimanenti (E) Boundaries of financial reporting, and Presentation and Disclosure, (F) Purpose and status of the framework e (G) Application of the framework to not-for-profit entities, devono ancora iniziare.

In modo molto più sommesso, anche in Europa qualcosa è già mosso. L'EFRAG già dal 2006 ha avviato nell'ambito delle sue iniziative pro-attive, a lanciare un progetto di Framework condiviso con il Conseil National de la Comptabilité francese, conclusosi nel 2009 con un Discussion paper, che in qualche modo ha cercato di influenzare lo sviluppo del nuovo Framework IASB. A febbraio 2010 ha pubblicato un altro documento circa la definizione 
di attività, assieme all'Autorité des Normes Comptable ed un documento sul criterio di contabilizzazione dei ricavi. Nel giugno 2011 è poi iniziato in seno all'EFRAG un progetto volto a capire come concretamente i bilanci sono impiegati dagli utenti.

La Commissione Europea direttamente non ha mai sviluppato un proprio Framework. Si potrebbe osservare che in qualche modo una specie di Framework sia già contenuta nelle Direttive Contabili (la IV del 1978 e successive modifiche), tenuto conto che tale documento stabilisce alcuni basilari postulati di bilancio, oltre al principio generale del quadro fedele ("true and fair view"). Ma sono assenti dalla direttiva alcuni requisiti fondamentali per quanto riguarda la definizione di reddito, di costo/ricavo, di attività/passività e di patrimonio netto, oltre all'assenza di tutta la parte concernente gli utenti del bilancio. Anche nelle bozze di riforma delle Direttive contabili che stanno circolando in questi mesi, non emergono tentativi di impostazione di un vero e proprio Framework europeo.

E in Italia? Nel nostro Codice Civile e nei principi contabili degli organismi professionali (ora principi contabili dell'OIC) un Framework nel senso sopra indicato non c'è mai stato. Il Codice si limita a riprendere i postulati della IV Direttiva CEE mentre il documento n. 11 del CNDC-CNR, poi ripreso dall'OIC, riprende molto da vicino il Framework IASB per quanto riguarda lo scopo del bilancio e i fabbisogni degli utenti, ma trascura alcune parti importanti, tra cui, in primis, i concetti di reddito, costo, ricavo, attività e passività, $i$ criteri generali di contabilizzazione e valutazione. Non vi sono peraltro dei progetti specifici italiani sul punto, anche considerando la natura ormai etero-diretta delle regole contabili vigenti nel nostro Paese (Commissione Europea o principi internazionali, in funzione della quotazione o meno delle imprese).

In questo quadro a chi scrive sembra opportuno anzitutto che anche il mondo universitario contribuisca al dibattito. Nel 2009 un gruppo di accademici statunitensi ha provveduto in seno all'American Accounting Association ad approvare un framework (Alternative Conceptual Frameworks for Financial Reporting).

Per noi Europei e soprattutto per noi italiani un progetto del genere sarebbe molto opportuno. In primo luogo servirebbe per definire meglio le nostre radici. Chi scrive, come molti altri colleghi, si è formato sui testi della migliore tradizione economico-aziendale italiana, dove di bilancio si è parlato molto. Tuttavia quello che forse è mancato a tali Maestri o forse alle loro Scuole è stato il condensare tale rilevante corpus teorico in un documento di venti-trenta pagine che potesse fungere da chiara guida per la prassi operativa e da sintesi efficaci per i giovani allievi. Questo lavoro finora è mancato e non sarebbe male che, sia pur tardivamente, venisse sviluppato.

Al tempo stesso è anche vero che l'evoluzione delle realtà aziendali ha introdotto da tempo nuove e molto rilevanti questioni che un Framework dovrebbe affrontare. 
Anzitutto sembra opportuno ritornare sulle finalità/funzioni del bilancio (funzione come rendiconto degli amministratori, funzione informativa, a sua volta differenziabile in informazione per l'interno o per l'esterno), per capire se tali funzioni oggi possano essere efficacemente assolte con un unico documento (la famosa questione del bilancio "unico"). Ormai nei Framework IASB e FASB si parte dall'assioma della finalità del bilancio come informativa per l'esterno e si deriva la funzione di stewardship o di informazione per l'interno come sottoprodotto della prima. Siamo tutti convinti che ciò sia la migliore strada?

Nella pratica accade che il modello di azienda al quale si fa riferimento condiziona molto la funzione del bilancio. Un bilancio per una società quotata assolve certo una funzione informativa più importante di quanto accada per una non quotata, ed all'interno di queste ultime, la dimensione aziendale pure gioca un certo ruolo. Un bilancio per una piccola impresa (ammesso che si possa definirla in modo chiaro) coinvolge certo meno stakeholder di una grande impresa.

Inoltre, nei framework IASB e FASB finora esistenti si parte dal chiaro presupposto di una user primacy (quella degli investitori esterni a titolo di capitale, come nuovi potenziali soci), espressione di un modello di azienda tipico della grande corporation e si assume che i bisogni informativi degli altri utenti siano comunque soddisfatti se sono soddisfatti gli interessi di tali investitori, ritenuti, a torto o a ragione, più sofisticati. È giusto che sia così? Le banche finanziatrici rivolgono al bilancio la stessa domanda informativa di un fondo comune di investimento? Chi legge realmente il bilancio? E cosa legge del bilancio? La questione è immensa ed apre la strada ad una indagine approfondita, estesa ed articolata, volta a comprendere gli effettivi fabbisogni informativi degli utenti. E nella recente letteratura sorgono molti interrogativi su quanto la comprensione dei fabbisogni informativi sia stata realmente considerata da parte degli organismi normativi ${ }^{1}$.

In relazione alla comprensione della effettiva domanda informativa, si aprono altri interrogativi di fondo per il framework. Cosa è un bilancio?/Quali sono i suoi confini? Se prescindiamo dai prospetti contabili, si tratta di capire se il Framework deve applicarsi anche alla relazione sulla gestione ed ai prospetti informativi che possono essere anche formalmente sganciati dal bilancio stesso, ma che formano assieme ad esso un unico pacchetto informativo (bilancio dell'intangibile o del capitale intellettuale, bilancio ambientale, bilancio di sostenibilità, bilancio sociale e chi più ne ha più ne metta). L'inclusione o meno di tali prospetti o di tali ambiti informativi in un framework dovrebbe per logica discendere dalla definizione del concetto di risultato (perfomance) aziendale che gli utenti desiderano conoscere.

1. Joni J. Young, Making up users, Accounting, Organizations and Society, vol. 31 (2006), pp. 579-600. 
Inoltre, a complicare il quadro, non si deve sottacere anche la rilevanza dei canali di comunicazione. Se gli investitori delle società quotate in borsa reagiscono alle informazioni circa redditi e capitale quando sono comunicati i dati consuntivi dell'esercizio nel mese di gennaio/febbraio tramite presentazioni o conference calls, ha senso continuare a improntare il Framework solo sul bilancio inteso come documento a sfondo contabile oppure avrebbe più senso affrontare la questione dell'informazione contabile in senso ampio, per includere i vari momenti/canali di comunicazione con l'esterno?

Insomma, ci sono questioni di fondo che andrebbero finalmente affrontate. Certo, è un lavoro duro... Ma qualcuno lo deve pur fare!

Pertanto, parafrasando Kennedy, cari studiosi non chiedetevi che cosa potrà fare il Framework per voi, ma che cosa potrete fare voi per il Framework. 